\title{
La aplicación de la terapia de aceptación y compromiso en dos casos de evitación experiencial*
}

\author{
The Application of ficceptance and Commitment Therapy in Two Cases \\ of Experiential fuoidance \\ f aplicação da terapia de aceitação e compromisso em dois casos \\ de evitação experiencial
}

\author{
Sergio Ribero-Marulanda, ${ }^{* *}$ Lina Agudelo-Colorado ${ }^{* *}$ \\ Fundación Universitaria Konrad Lorenz, Bogotá, Colombia.
}

Doi: dx.doi.org/10.12804/ap134.1.2016.03

\section{Resumen}

El objetivo principal de este estudio fue identificar la efectividad de la terapia de aceptación y compromiso en dos mujeres con el trastorno de evitación experiencial mediante el diseño y la implementación de una estructura de intervención psicoterapéutica en la ciudad de Bogotá, con un diseño de caso único de tipo $\mathrm{AB}$ con seguimiento. Se encontró que los procesos de inflexibilidad psicológica que causan malestar en las personas están sujetos al trabajo clínico con una respuesta positiva, ya que desde la terapia de aceptación y compromiso y las técnicas que desde allí se proponen, las participantes empezaron a dirigir su vida hacia sus valores y relaciones deseables libremente.

Palabras clave: análisis de la conducta; evitación experiencial; valores; teoría de marcos relacionales y terapia de aceptación y compromiso.
Abstract

The main objective of this study was to identify the effectiveness of the Acceptance and Commitment Therapy in two women with experiential avoidance disorder through the designed structure and implementation of a psychotherapeutic intervention in Bogotá with a single case design of type $\mathrm{AB}$ with follow-up. We found that the processes of psychological inflexibility, causing discomfort in people, are subject to clinical work with a positive response, since from the Acceptance and Commitment Therapy and techniques from there proposed, the participants began to turn their life to desirable values and relationships freely.

Keywords: analysis of behavior; experiential avoidance; values; relational frame theory and acceptance and commitment therapy.

* El presente documento es el producto de la investigación realizada en el proceso de prácticas de psicología clínica de los autores en la Unidad de Orientación y Asesoría Psicológica de la Universidad Cooperativa de Colombia.

** Sergio Ribero-Marulanda, psicólogo. Mg.; Lina Agudelo-Colorado, psicóloga.

La correspondencia relacionada con este artículo debe ser dirigida a Sergio Ribero-Marulanda Correo electrónico: ribero.sergio. sr@gmail.com, o a Lina Agudelo-Colorado. Correo electrónico: linamas1@hotmail.com

Cómo citar este artículo: Ribero-Marulanda, S. \& Agudelo-Colorado, L. (2016). La aplicación de la terapia de aceptación y compromiso en dos casos de evitación experiencial. Avances en Psicología Latinoamericana, 34(1), 29-46. doi: dx.doi.org/10.12804/ apl34.1.2016.03 


\section{Resumo}

O objetivo principal deste estudo foi identificar a efetividade da Terapia de Aceitação e Compromisso em duas mulheres com o Transtorno de Evitação Experiencial através do desenho e implementação de uma estrutura de intervenção psicoterapêutica na cidade de Bogotá, com um desenho de caso único de tipo $\mathrm{AB}$ com seguimento. Encontrou-se que os processos de inflexibilidade psicológica, que causam mal-estar nas pessoas, estão sujeitos ao trabalho clínico com uma resposta positiva, devido a que desde a Terapia de Aceitação e Compromisso e as técnicas que desde ali se propõem, as participantes começaram a dirigir sua vida a seus valores e relações desejáveis livremente.

Palavras-chave: análise da conduta; evitação experiencial; valores, teoria de marcos relacionais; terapia de aceitação e compromisso.

El sufrimiento, como parte de la vida humana, tiene implicaciones verbales que la comunidad ha instaurado en un contexto lingüístico en el que sentirse "triste", "fracasado" o "temeroso" se ha relacionado con estados "negativos" que llegan a juzgarse como inaceptables. Así es como las personas intentan controlar estos sentimientos con el fin de buscar un estado de "satisfacción" o "felicidad". Dentro de los repertorios de control, los seres humanos emiten comportamientos adaptativos de evitación o escape ante situaciones que les imperen contacto con estímulos considerados "negativos", según su historia individual; sin embargo, el exceso de control de las experiencias privadas particulares (sensaciones corporales, pensamientos o recuerdos) y de los contextos que dan lugar a estos eventos privados es lo que define el trastorno de evitación experiencial (TEE) (Bach \& Hayes, 2002; Hayes, Wilson, Gifford, Follette \& Strosahl, 1996; Luciano, Gutiérrez \& Rodríguez,2005; Luciano \& Hayes, 2001; Wilson \& Luciano, 2002).

El TEE se puede entender a partir de la generación de relaciones arbitrarias de la conducta verbal o de repertorios conductuales necesarios para relacionarse socialmente (Ribes, Torres \& Martínez, 1996). Dichas relaciones arbitrarias se comprenden a partir del fenómeno de igualación a la muestra, en el cual, a partir del entrenamiento de dos relaciones, surgen nuevas relaciones con elementos que no han aparecido juntos (Sidman, 1971, citado en Valero \& Luciano, 1992).

De esta manera, en la medida en que las personas tienen lenguaje, se relacionan arbitrariamente con los eventos y responden no solo a las propiedades formales de los estímulos, sino a las funciones que dichos estímulos tienen dentro de la comunidad verbal de referencia (Wilson \& Luciano, 2002; Torneke, Luciano \& Valdivia, 2008; Barnes, Barnes, Smeets, Cullinan \& Leader, 2004). En este desarrollo conceptual surge la teoría de marcos relacionales como una perspectiva para entender la conducta verbal y la cognición, estudiando cómo el comportamiento relacional llega a ser extraído a través del mismo lenguaje (Barnes et al., 2004).

Teniendo en cuenta los anteriores estudios y la influencia de la sociedad en la creación de problemas psicológicos, surgen las terapias de tercera generación, que identifican la conducta en función de las variables del contexto (Luciano \& Valdivia, 2006, citados en Lozano \& Caycedo, 2008). Entre estas se encuentra la terapia de aceptación y compromiso (ACT por su sigla en inglés), orientada hacia metas centradas en la activación y la aceptación psicológica, cuyo fin último es dotar al individuo de repertorios conductuales flexibles y amplios, además de trabajar sobre la conciencia del momento presente, los valores, sin buscar la eliminación, la lucha o la disminución de los eventos privados (pensamientos o sentimientos ligados específicamente con el sufrimiento del ser humano) (Lozano \& Caycedo, 2008; Luciano \& Valdivia, 2006; Hayes, Luoma, Bond, Masuda \& Lillis, 2006; Kohlenberg, Hayes \& Tsai, 1993; Hayes, Masuda, Bissett, Luoma \& Guerrero, 2004; Hayes, 2004).

De esta manera, para llevar a cabo un proceso terapéutico que tenga eficacia y profundidad en el 
TEE, la ACT ofrece un conjunto de herramientas clínicas que permiten desmontar los procesos de evitación y de seguimiento de reglas (Kohlenberg, Tsai, Ferro, Valero, Fernández \& Virués-Ortega, 2005 , p. 350), buscando que los cambios en el contexto verbal y de interacción dentro de la sesión produzcan efectos positivos en repertorios que estén orientados a acciones comprometidas fuera de sesión.

La ACT, a través de seis componentes, busca que el ser humano: (1) identifique y abandone esas estrategias de control de los eventos internos, (2) que observe las experiencias sin tener que etiquetarlas y llevarlas a ser literalmente ciertas y (3) se centre en conductas orientadas a obtener resultados valiosos, para crear de esta manera acciones flexibles y eficaces (Bach \& Hayes, 2002; Eifert, Forsyth, Arch, Keller \& Langer, 2009; Hayes et al., 2006, citados en Russo \& Forsyth, 2013; Valdivia, Sheppard \& Forsyth, 2010; Wilson, Bordieri, Flynn, Lucas \& Slater, 2010).

Estos componentes se agrupan en un modelo de psicopatología y salud psicológica llamado Hexaflex, creado por Wilson (2007). Dentro de la ACT se hacen cambios contextuales por medio de metáforas, paradojas y ejercicios experienciales, que enseñan al cliente "que el dolor psicológico y el malestar son facetas ubicuas e inevitables de la vida, y por lo tanto no pueden ser totalmente evitadas o eliminadas" (Russo \& Forsyth, 2013, pp. 54 y 55), pues los valores a largo plazo y las áreas significativas de la vida de una persona estarían cubiertas de los esfuerzos por evitar las experiencias negativas, y así se abandonarían sus valores y propósitos (Prevedini, Presti, Rabitti, Miselli \& Moderato, 2011).

Diferentes estudios han demostrado la efectividad de la ACT, como lo indica Ruiz (2010), quien afirma que es eficaz en un amplio rango de problemas como el dolor crónico, la depresión, el trastorno obsesivo-compulsivo, el trastorno de ansiedad generalizada, entre otros, ya que los protocolos basados en la aceptación son más efectivos que los que se basan en el control. En este estudio se hace una revisión de investigaciones realizadas por Hayes et al. (2006), Hayes et al. (2004), Gaudiano (2009) y Powers, Zum Vörde Sive Vörding y Emmelkamp (2009), las cuales demuestran que la intervención en ACT tiene mayor efecto en las conductas problema que aquellas personas que están en grupo control, las que están sometidas a condición placebo, grupos en lista de espera u otros tratamientos, en estudios de ensayos de control aleatorio desde un análisis cualitativo y cuantitativo.

Teniendo en cuenta la revisión teórica realizada, la presente investigación busca aplicar la ACT en dos mujeres con evitación experiencial, para evidenciar los cambios conductuales y la flexibilidad psicológica como resultado de la intervención propuesta. La investigación dentro de este campo resulta ser útil para el desarrollo profesional de los psicólogos clínicos, ya que permite, por un lado, abordar de una manera efectiva los procesos relacionados con el TEE y, por otro, generar estrategias de intervención acordes y eficaces. Con el fin de que las clases funcionales del TEE lleguen a disminuir $\mathrm{y}$, además, ir aumentando la evidencia empírica respecto a este tipo de terapias basadas en el contextualismo funcional (Kohlenberg et al., 2005), el presente trabajo le va a permitir a los psicólogos con poca experiencia en el modelo de ACT tener una amplia gama de herramientas y un ejemplo de trabajo sistemático para abordar otro tipo de problemas clínicos desde ACT. Como indica Rey (2008), la ACT permite intervenir sobre conductas que ponen en riesgo la salud, como conductas sexuales de riesgo o el consumo de alcohol, por el trabajo en los componentes de compromiso personal inherente a la misma terapia.

\section{Método}

\section{Diseño}

Se usó un diseño de caso único, ya que posibilita que el individuo sea su propio control durante el 
proceso terapéutico (García-Celay \& León, 2005). El tipo de diseño fue $\mathrm{AB}$ con seguimiento, donde $A$ es la identificación de la conducta, previa a la implementación del tratamiento, y $B$ es la aplicación del tratamiento, teniendo presente el cambio en las variables dependientes y, finalmente, el seguimiento con el cual se buscó identificar la estabilidad de la intervención con el paso del tiempo. Este tipo de diseño tiene ciertas limitaciones: (1) los resultados no tienen una fuente de comparación, pues solo pueden compararse intracaso y nunca entre casos, y (2) existe una gran variabilidad de los datos que se obtienen en la línea base; por ello la predicción y conclusión acerca del tratamiento puede ser más difícil (Roussos, 2007). La investigación se realizó bajo un corte cuasi experimental, pues se encontraron ciertas limitaciones y variables extrañas, como continuidad del proceso, tiempo entre sesiones, situaciones particulares en la vida de las consultantes y asistencia continúa a las sesiones, que no se lograron controlar del proceso (Montero \& León, 2002).

\section{Participantes}

La muestra estuvo conformada por dos mujeres que asistieron a la Unidad de Orientación y Asesoría Psicológica de la Universidad Cooperativa de Colombia, sede Bogotá, y ambas participaron de manera voluntaria en la investigación. La edad de la primera participante era 49 años, y la de la segunda, 36 años. Las dos mujeres tenían hijos y vivían en el estrato socioeconómico 3 (medio-bajo), donde se alberga la media poblacional de Bogotá.

\section{Definición de variables}

Variable independiente. Intervención psicológica basada en los componentes de la ACT. Esta variable corresponde a la implementación de los seis componentes de la ACT, los cuales trabajan en el espectro flexibilidad-inflexibilidad psicológica (Hayes, Strosahl, Bunting, Twohig \& Wilson,
2004). Dentro de la investigación se tuvo control del proceso de tratamiento mediante la creación e implementación de una estructura de intervención, en la cual se aplicaron ejercicios experienciales, metáforas y ejercicios de mindfulness que permiten en sesión dar el contexto verbal para aumentar la flexibilidad psicológica de las participantes. Esta propuesta fue adaptada de la estructura propuesta por Bond y Hayes (2002).

Variables dependientes. Trastorno de evitación experiencial. Se entiende como todas aquellas acciones que involucran tanto la conducta verbal como la no verbal, que cumplen la función de evitar entrar en contacto con sensaciones, pensamientos, emociones o verbalizaciones que son desagradables para ellas teniendo en cuenta su contexto verbal. Estas acciones se identificaron a partir del análisis ideográfico y funcional de cada caso. Esta variable se midió por medio de los componentes del Hexaflex a partir de las verbalizaciones hechas por cada participante durante las sesiones programadas y por la aplicación del Cuestionario de Aceptación y Acción (AAQ) II-9, en dos ocasiones.

\section{Instrumentos}

Cuestionario de Aceptación y Acción II-9 (Barraca, 2004). Este cuestionario evalúa en qué medida las personas, ante sus eventos privados, asociados con el malestar psicológico, logran aceptarlos y mantener presente sus metas y valores, orientando sus acciones hacia éstas (Hayes et al., 2004). El cuestionario AAQ-II-9 consta de nueve ítems en escala tipo Likert que ha sido validado en población española (Barraca, 2004). Altas puntuaciones indican menos aceptación, es decir, mayor evitación experiencial. Esta prueba cuenta con una consistencia interna $\alpha=.74$; al realizarse la adaptación del inglés al español, se encontró que la media de la prueba en grupo clínico fue de 44.71 con una desviación de 8.42 y para el grupo no clínico la media fue 34.61 y la desviación fue de 5.41. 
Al realizar la validez de constructo, siguiendo el criterio de Barraca (2004), se obtuvieron tres dimensiones: el factor 1, denominado desbordamiento emocional, que estuvo conformado por los ítems 3, 5, 7, 8 y 9; el factor 2, llamado evaluación inadecuada de los problemas de la vida y que estuvo conformado por los ítems 2 y 6 , y el factor 3 , respuesta ineficaz a los problemas de la vida, conformado por los ítems 1 y 4 . Dado que existe una adaptación al español del AAQ se decidió usarla en la presente investigación y se determinó utilizar las tres dimensiones propuestas por dicho autor. Este instrumento se aplicó pretest y postest, con el fin de evidenciar el cambio en la "actitud de evitación".

Hexaflex (Wilson, 2007). Este instrumento lo completaron cada uno de los dos terapeutas al finalizar las sesiones terapéuticas, lo que permitió evaluar los seis procesos de flexibilidad psicológica en las participantes. La escala que permite la clasificación de cada componente se encuentra de 1 a 5 , donde 1 es la puntuación mínima y 5 es la puntuación máxima de flexibilidad psicológica. Para obtener mayor sistematicidad y alcanzar mayor control en la calificación del Hexaflex, los terapeutas llegaron al acuerdo de calificar cada componente, teniendo en cuenta las verbalizaciones en relación con cada componente durante las sesiones; de esta forma, sobre el $100 \%$ de verbalizaciones emitidas en sesión por cada participante que hacían referencia a lo que ocurría y lo que ocurría en el contexto natural de cada una, se calificó 1 si solo el 15\% de las verbalizaciones estaban orientadas a dicho componente, 2 con un $30 \%$ de verbalizaciones, 3 con un $40 \%, 4$ con un $50 \%$ y 5 para un $65 \%$ de las verbalizaciones. En este punto es importante aclarar que existía una correspondencia decir-hacer, que permitió dar cuenta de la actuación real de las participantes y así se calculó el total de las verbalizaciones y el porcentaje por cada componente. Destacar finalmente la relevancia del Hexaflex como un instrumento de medida efectivo en la ACT, en relación con su validez, permite obtener medidas fiables en cuanto a la flexibilidad psicológica.

Así, el proceso estuvo guiado por una estructura de intervención, donde se especificaba sesión a sesión las actividades que se iban a realizar. Esta estructura se basó en la ACT, y fue construida a partir de las necesidades propias de las participantes. Para su elaboración se retomó el esquema general de la propuesta por Bond y Hayes (2002).

Los resultados obtenidos en la investigación propuesta por Suárez-Aguirre, Luciano y Ruiz (2010) muestran la importancia de la realización de estructuras o protocolos breves de intervención en la aplicación de ACT, que vayan dirigidos a facilitar que los participantes den cuenta de sus pensamientos y sensaciones relacionadas con el malestar; mientras continúan centrados en sus tareas y valores, es decir, sin comportarse de manera literal a ellos. De este modo, aunque los participantes experimenten malestar, se esperaba un aumento de sus acciones orientadas a sus respectivos valores.

Es importante destacar que la estructura de intervención estuvo compuesta por ciertas fases expuestas en el libro de Hayes, Strosahl y Wilson (1999), como desesperanza creativa, control como el problema, valores y acción comprometida. Se comenzó con una medición de línea de base durante las primeras cuatro sesiones; posteriormente, se aplicó la estructura de intervención.

Finalmente, para lograr el análisis funcional se partió de las verbalizaciones que las participantes daban en sesión. Estos datos se organizaron teniendo en cuenta la Guía de formulación de caso clínico (Muñoz \& Novoa-Gómez, 2010), sistema que permitió ordenar esta información procedente de la evaluación y facilitó desarrollar un plan de intervención dirigido a las necesidades de las participantes.

\section{Procedimiento}

En la fase inicial, los dos terapeutas contactaron a las participantes a través de una llamada 
telefónica, después de que ellas solicitaran previamente el servicio de psicología en la Unidad de Orientación y Asesoría Psicológica de la Universidad Cooperativa de Colombia. Después de las cuatro primeras sesiones de evaluación y al identificar puntos en común en las dos participantes, específicamente repertorios orientados a la evitación experiencial, se les preguntó si deseaban hacer parte de la investigación presente. De esta manera se inició la intervención voluntaria y se formalizó su participación mediante el consentimiento informado y autorización de la grabación en video de todas las sesiones terapéuticas, según la ley del psicólogo (Congreso de la República, 2006).

Estas primeras cuatro sesiones configuraron la fase evaluativa y la línea base del estudio. En ellas se adelantó la evaluación ideográfica de los repertorios de cada participante, a través de una entrevista semiestructurada orientada a la realización del modelo de formulación de caso clínico (Muñoz \& Novoa-Gómez, 2010).

La aplicación de estos instrumentos permitió obtener datos acerca de los repertorios conductuales de cada una de las participantes y su relación con su vida. Las participantes completaron el AAQ-II durante la sesión de evaluación y de seguimiento. Los datos del Hexaflex y sus respectivos análisis se llevaron a cabo al finalizar cada sesión por parte de los terapeutas, a partir de las verbalizaciones de las consultantes hechas en sesión, que daban cuenta de acciones comprometidas y cambios concretos fuera de sesión. Cuando se logró una estabilidad en las puntuaciones del Hexaflex en las primeras cuatro sesiones, se pasó a la fase de intervención.

Las sesiones de intervención (siete sesiones) se iniciaron después de una breve retroalimentación a las consultantes, explicándoles lo hallado durante la evaluación. Cada sesión duró 60 minutos, con periodicidad semanal y, en ocasiones, quincenal. $\mathrm{Al}$ iniciar las sesiones terapéuticas se retomó parte de lo tratado en las sesiones anteriores y su impacto en el contexto natural de cada una, indagando a su vez temas referentes a las tareas que se les habían mandado, y en caso de no haberlas realizado, se tomaban unos minutos para hablar sobre ello.

La estructura de intervención no se presentó de manera rígida en el orden de cada sesión, pues dependía de los repertorios puntuales de cada participante; sin embargo, al finalizar el proceso de intervención, sí se llevó a cabo en su totalidad con cada una de ellas.

La estructura contaba con: (1) ejercicios de mindfulness, (2) aplicación de metáforas y (3) ejercicios experienciales. Los ejercicios de mindfulness estuvieron orientados a generar en las participantes la capacidad de vivir el aquí y el ahora sin generar juicios de valor, realizando ejercicios de respiración, descripción, observación y conciencia del propio cuerpo. Por otro lado, las metáforas que se utilizaron fueron: "El hombre en el hoyo", con el fin de generar un estado de desesperanza creativa. "El jardín”, “el epitafio" y la del "Conductor del autobús y los pasajeros" para que las participantes orientaran sus acciones hacia sus valores tomando perspectiva de su propia experiencia. La metáfora de "los números" les permitía a las participantes experimentar el aspecto aditivo de la historia y el efecto que esta tiene en las respuestas actuales y, finalmente, la metáfora de "las llaves" para que las participantes aprendieran a "estar abiertas a los pensamientos y sentimientos que más se temen sin tener límites en tanto que permiten hacer los recorridos vitales que se quieren hacer" (Wilson \& Luciano, 2002).

Finalmente, los ejercicios experienciales se utilizaron con el propósito de confrontar a las participantes frente a experiencias difíciles para ellas y situaciones previas de fracaso, para ser contrastado con esos valores que se han generado hasta el momento. Estos ejercicios experienciales se crearon y se usaron a partir de la propia historia de las participantes y sus dificultades particulares. La finalización de la intervención se dio por la tendencia positiva en las puntuaciones del Hexaflex, lo que dio cuenta de las mejorías en sesión y el incremento de conductas en el contexto natural 
de las participantes orientadas a la flexibilidad psicológica, según su reporte.

Posteriormente, se llevó a cabo la fase de verificación postratamiento o seguimiento en las últimas dos semanas y a los tres meses, después de finalizada la intervención, para completar un total de diez sesiones de tratamiento. En esta parte se aplicaron nuevamente cada uno de los instrumentos; se les preguntó a las participantes por sus áreas de ajuste (trabajo, familia, amigos, entre otras) y por las situaciones difíciles que les han ocurrido y que han hecho al respecto, con el fin de identificar el mantenimiento de los cambios dentro de las sesiones, y se indagó acerca de las acciones que han llevado a cabo para alcanzar sus valores. Según el criterio del terapeuta se incluyeron metáforas que servían para afianzar el cambio conductual y se realizaron ejercicios de mindfulness como los que se tuvieron en cuenta durante la fase de intervención. Es importante aclarar que la fase de seguimiento se llevó a cabo como lo indica el protocolo de Bond y Hayes (2002), es decir, una sesión de seguimiento a los tres meses; sin embargo, para mayor control se agregaron dos sesiones más de seguimiento, una ocho días después de culminada la intervención y otra a los quince días.

Por último, se analizaron los resultados intrasujeto a través de la comparación de resultados obtenidos para cada instrumento (AAQ-II y Hexaflex).

\section{Resultados}

Los datos obtenidos a través de medidas continuas en las fases de evaluación (línea base), intervención y seguimiento se exponen a continuación. Los resultados intrasujeto se describen en las siguientes figuras, haciendo referencia a cada una de las etapas del proceso terapéutico y detallando los resultados del AAQ-II, los componentes del Hexaflex y sus puntuaciones por sesión. Los resultados del Hexaflex y sus análisis se lograron uniendo los componentes en parejas, como se propone en el libro de Dahl, Stewart, Kaplan y Martell (2013).

\section{Participante I}

Motivo de consulta. El proceso inicio por voluntad de la participante, la cual indicó: "Vengo porque quiero tener una vida tranquila", "todo lo hago por salud mental".

Hipótesis explicativa. Presentaba un patrón de evitación caracterizado por sumisión y el consentimiento de malos tratos por parte de sus hijas como: reproches por la separación con su esposo, salidas a la tienda o gustos personales como ropa, paseos y amigos, ante lo cual la participante responde agachando la cabeza, pidiendo disculpas, pidiendo permiso y aceptando la opinión de los demás por encima de sus sentimientos y puntos de vista. Además, mostraba constantes pensamientos acerca de que lo que hace puede estar mal y que los demás (hijas, familiares o vecinos) pueden estar diciendo o pensando sobre lo que ella quisiera hacer. Según se hipotetizó, esto se adquirió a través de la conducta gobernada por reglas durante toda su historia de desarrollo, por ejemplo: "Ser una buena madre es estar siempre con sus hijos sin importar nada", "uno debe aguantar todo por sus hijos", "una buena madre mantiene la familia unida" y "El matrimonio es para toda la vida". Y se mantenía por la derivación de reglas y la coherencia contextual de estas dentro de su comunidad verbal de referencia, ya que todas las personas cercanas en su contexto validaban y reforzaban este tipo de comportamientos y por el refuerzo negativo, ya que al emitir esas conductas, disminuía la probabilidad de emisión de reproches y malos tratos (gritos e insultos por parte de sus hijas).

A continuación se presentan los resultados obtenidos por la participante 1, durante las fases de línea de base, tratamiento y seguimiento en el caso del Hexaflex, y en el caso del AAQ-II se muestran los resultados de línea de base y fase de seguimiento.

AAQ-II. La figura 1 corresponde al análisis global de los resultados encontrados en la aplicación 
pretest y del postest del AAQ-II de la participante 1. La puntuación total general del pretest es de 42 puntos. Esta indica que la consultante no puede ser considerada población clínica, en cuanto al TEE con la medida usada, pues la medida clínica es de 44,71. Esto no quiere decir que no presente un problema de evitación experiencial. Posterior a la intervención, se identifica cómo la participante, en cada uno de los factores (desbordamiento emocional, evaluación inadecuada de los problemas de la vida y respuestas ineficientes para los problemas de la vida), obtuvo puntuaciones más bajas. En el primer factor se evidencia disminución de 11 puntos (47.82\%) de respuestas asociadas a la percepción de incapacidad de hacer frente a las responsabilidades y la aceptación de sus sentimientos. En el segundo factor se observa una disminución de 3 puntos $(30 \%)$, lo que indicaría el aumento de respuestas encaminadas a la solución de problemas de la vida diaria y a la aceptación de los eventos privados sin dejar que estos controlen su conducta. Finalmente, en el tercer factor se encontró una disminución de 2 puntos $(22.22 \%)$, lo que evidencia una percepción, por parte de la participante, de respuestas eficaces para los problemas de la vida diaria.

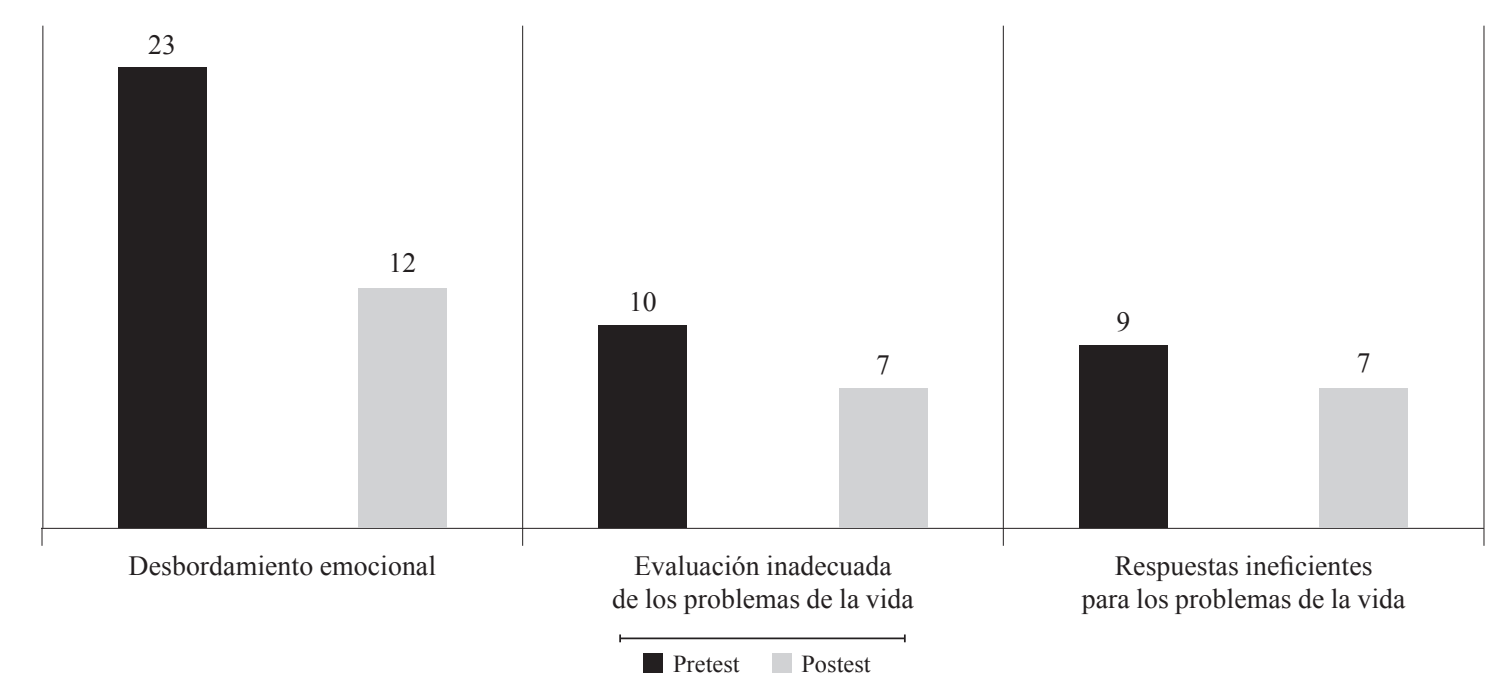

Figura 1. Puntuaciones obtenidas pretest y postest por la participante 1 en el AAQ-II
Se observa mayor cambio favorable en el ítem 5 ("mis sentimientos no me dan miedo"), seguido de los ítems 6 y 4 , con 4 y 3 puntos menos, respectivamente, en relación con la medición pretest. Por otro lado, se observa un pequeño aumento (1 punto) en el ítem 2 ("Muchas veces me descubro fantaseando sobre cosas que he hecho y que haría de forma distinta si tuviera otra oportunidad").

Hexaflex. En la figura 2 se muestran los resultados obtenidos por medio del Hexaflex y se observa cómo en la línea base se mantiene una regularidad de calificaciones en todos los datos, al obtenerse puntuaciones bajas en los componentes de aceptación, defusión cognitiva, yo contexto y momento presente, que se mantienen en la evaluación con un punto, es decir, que solo el $15 \%$ de sus verbalizaciones daban cuenta de acciones concretas fuera de sesión orientadas a la flexibilidad psicológica de estas áreas. Durante la intervención, la calificación para los componentes de aceptación, yo contexto y momento presente se dan en un incremento progresivo desde la sesión 4 hasta la sesión 9 y se mantienen hasta el final de la intervención una puntación medio-alta (4 puntos). En la sesión 7 existió un descenso a una puntuación baja 


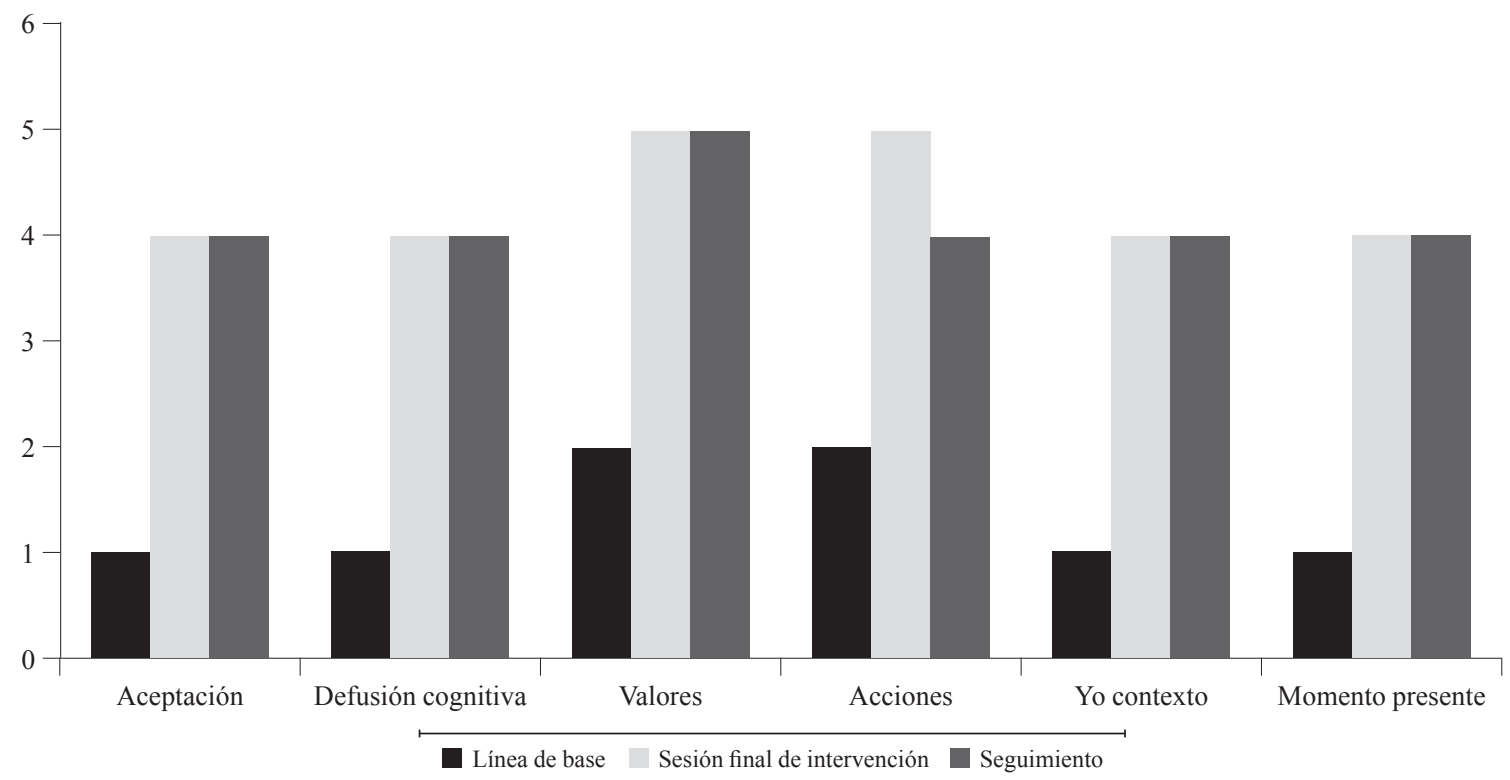

Figura 2. Puntuaciones obtenidas en la línea de base, al final de la intervención y en el seguimiento por la participante I en los componentes del Hexaflex

( 2 puntos) en todos los componentes, posiblemente relacionado con una fuerte discusión que tuvo con su hija y su familia. En la fase de seguimiento, estos cuatro componentes mantienen una regularidad de puntuaciones medias-altas en todas las sesiones con tendencia a mantenerse en el tiempo.

El incremento en la calificación de la defusión cognitiva fue más lento y se mantuvo en puntuaciones medias ( 3 puntos) durante casi todas las sesiones; sin embargo, presentó un incremento al finalizar la intervención hacia un puntaje de 4 puntos. En la fase de seguimiento llevada a los 8 y 15 días después de finalizada la intervención se evidencia una tendencia positiva a mantener la flexibilidad de estos componentes, al obtener puntuaciones medias-altas.

En relación con los componentes de valores y acciones comprometidas se puede observar que estas no inician en un puntuación de 1 como los anteriores componentes, ya que la participante 1 se dirigía con mayor facilidad a la creación de una vida con acciones bajo el dominio de direcciones valiosas. De ahí que durante la fase de intervención las calificaciones aumentaron progresivamente de la sesión 4 hasta la sesión 10, sin que la discusión familiar, destacada previamente, tuviera gran repercusión en su progreso. Finalmente, en términos de las sesiones de seguimiento, se observa una tendencia a mantener calificaciones medias-altas, que llegan a tener un pico positivo en el componente de valores en la última sesión de seguimiento.

\section{Participante 2}

Motivo de consulta. El proceso inició por voluntad de la participante, la cual indicó: "Tengo problemas con mi esposo, debido a mi intensa ira".

Hipótesis explicativa. Patrón de evitación caracterizado por pensamientos que están dirigidos a la búsqueda e identificación de las características que debe tener una pareja. Por ello, a lo largo de su vida ha mantenido relaciones conflictivas con sus parejas, donde están presentes gritos, insultos y la expresión de inconformidades. Finalmente, responde en función de esas relaciones verbales 
arbitrarias que ha instaurado a lo largo de su historia personal. Las posibles hipótesis frente al caso indican que se ha adquirido a través de la conducta gobernada por reglas como: "Una familia ideal debe estar conformada por papá, mamá e hijo", "En una familia ideal debe haber estabilidad económica y emocional" y "Si no tengo esposo fracaso", que ha aprendido durante toda su historia de desarrollo; además de la imitación de repertorios conductuales del padre ante situaciones similares. Dicho patrón conductual se mantiene por la derivación de reglas y la coherencia contextual de estas dentro de su comunidad verbal de referencia, ya que todas las personas cercanas en su contexto validaban y reforzaban este tipo de comportamientos.

AAQ-II. En la figura 3 se observan los resultados globales del pretest y postest del AAQ-II de la participante 2. Fue 29 la puntuación del pretest, que, al igual que la anterior participante, no puede ser considerada población clínica, en cuanto al TEE con la medida usada; sin embargo, sí se presenta un problema de evitación experiencial y la puntuación del postest es de 23 puntos. Los datos indican una disminución en los resultados en los tres factores. En el factor desbordamiento emocional, se evidenciaron mayores puntuaciones en el pretest, en relación con los otros factores, y tras la intervención, se observó un decremento del $16.66 \%$, lo que puede verse relacionado con la aplicación de los componentes de la ACT, que posiblemente llevaron a la disminución del miedo de la participante sobre sus sentimientos y la aceptación de las experiencias dolorosas en su vida. En el factor evaluación inadecuada de los problemas de la vida se evidencia una puntuación inicial de 7 puntos, y tras la intervención este valor disminuye a 6 puntos, es decir, aunque hubo un cambio en las percepciones de la participante acerca de ver sus acciones como inadecuadas ante los problemas de la vida, estas no son suficientes para que ella las acepte. Finalmente, en el factor respuestas ineficientes para los problemas de la vida, se pudo evidenciar una disminución del 50\% en la medida del postest, lo que indicaría una mejor capacidad para encaminar sus acciones hacia sus valores.

En particular se encuentra que los ítems 5 y 9 presentan mayor cambio favorable (mis sentimientos me dan miedo y ojalá pudiera borrar por arte de magia todas las experiencias dolorosas que he tenido en la vida, respectivamente); sin embargo, aunque en el resto de ítems se evidencia disminu-

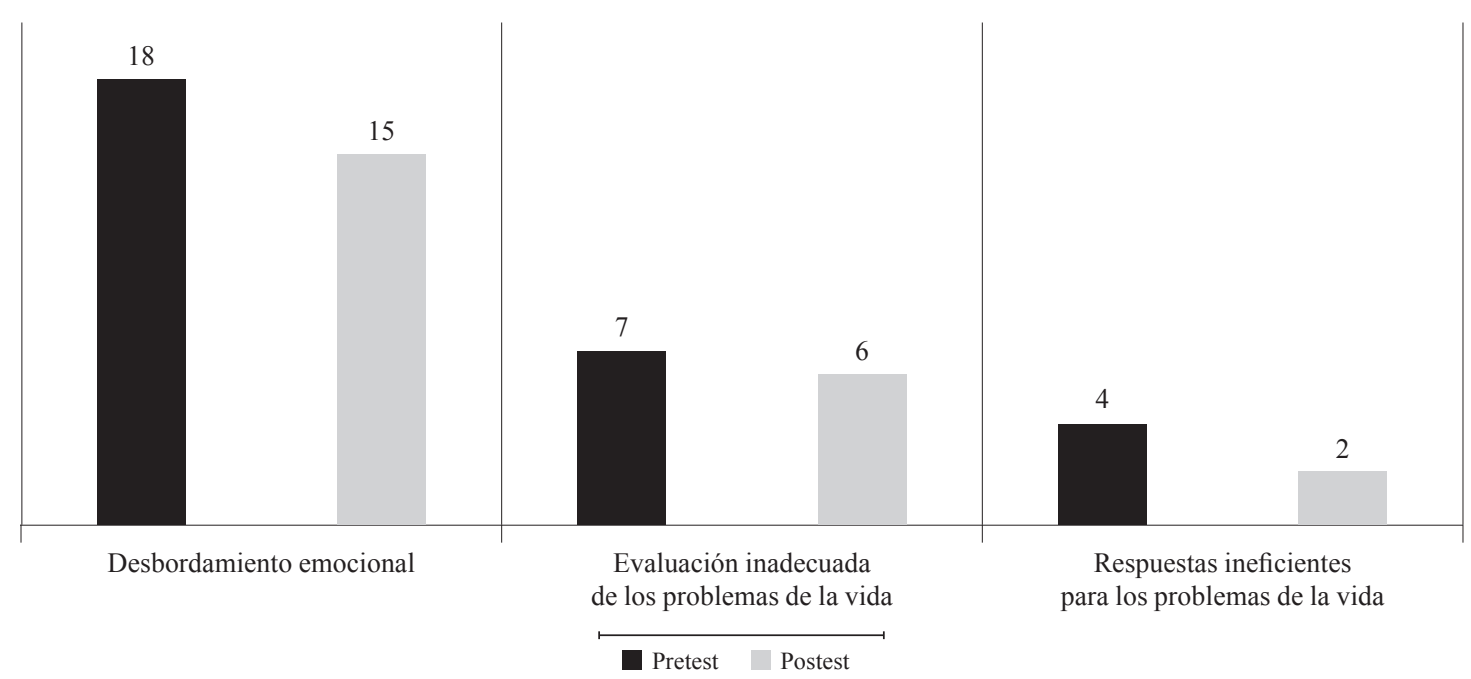

Figura 3. Puntuaciones obtenidas por la participante 2 durante el pretest y el postest en la aplicación del AAQ-II 
ción en las puntuaciones, los cambios evidenciados pretest/postest no sobrepasan 1 punto. Por otra parte, en el ítem 8 se ve un aumento de 1 punto. Adicionalmente, se observa el mantenimiento en las puntuaciones de los ítems 3, 6, y 7 .

Hexaflex. En la figura 4 se observa cómo en la línea de base se mantiene una regularidad en los datos, pues se obtuvo una puntuación baja en los valores del Hexaflex en todos los componentes (1 punto) durante las tres sesiones de evaluación, inclusive en la primera sesión de intervención. Los componentes de defusión cognitiva, acciones comprometidas, yo contexto y momento presente en las primeras sesiones de intervención 4 y 5 se mantienen en puntuaciones bajas, que aumentan solo desde la 6 hasta el final a puntuaciones medias en su calificación y se mantienen en puntuaciones medias hasta la última sesión de seguimiento, es decir, el repertorio de la participante 2 se caracterizó a lo largo de las sesiones por una constante fusión con sus pensamientos y emociones negativas que la hacían mantener juicios frecuentes, justificacio- nes o explicaciones de su conducta. Se dificultó la flexibilidad psicológica, pues la conducta estaba orientada a una preocupación o rumia constante, donde se mantiene el miedo al pasado y la incertidumbre al futuro, que le impide contacto con las contingencias presentes.

En el caso del componente de aceptación, aumentó progresivamente a lo largo de la intervención y llegó en la última sesión a su máximo valor de 5 puntos, es decir, que el $65 \%$ de sus verbalizaciones daban cuenta de acciones que estaban orientadas a describir y vivir los eventos difíciles en sesión y fuera de ella, sin necesidad de modificarlos o evitarlos y mantener sus puntuaciones en la fase de seguimiento en puntajes altos. Aunque el componente de valores varió en la línea de base de 1 a 2 puntos, desde la implementación de la intervención se vio una modificación conductual reflejada en el componente de valores que aumentó gradual y positivamente hacia la flexibilidad psicológica y demostró la elección de direcciones valiosas, que igualmente llegaron a 5 puntos de calificación. No obstante, cabe indicar que a pesar de su elección de

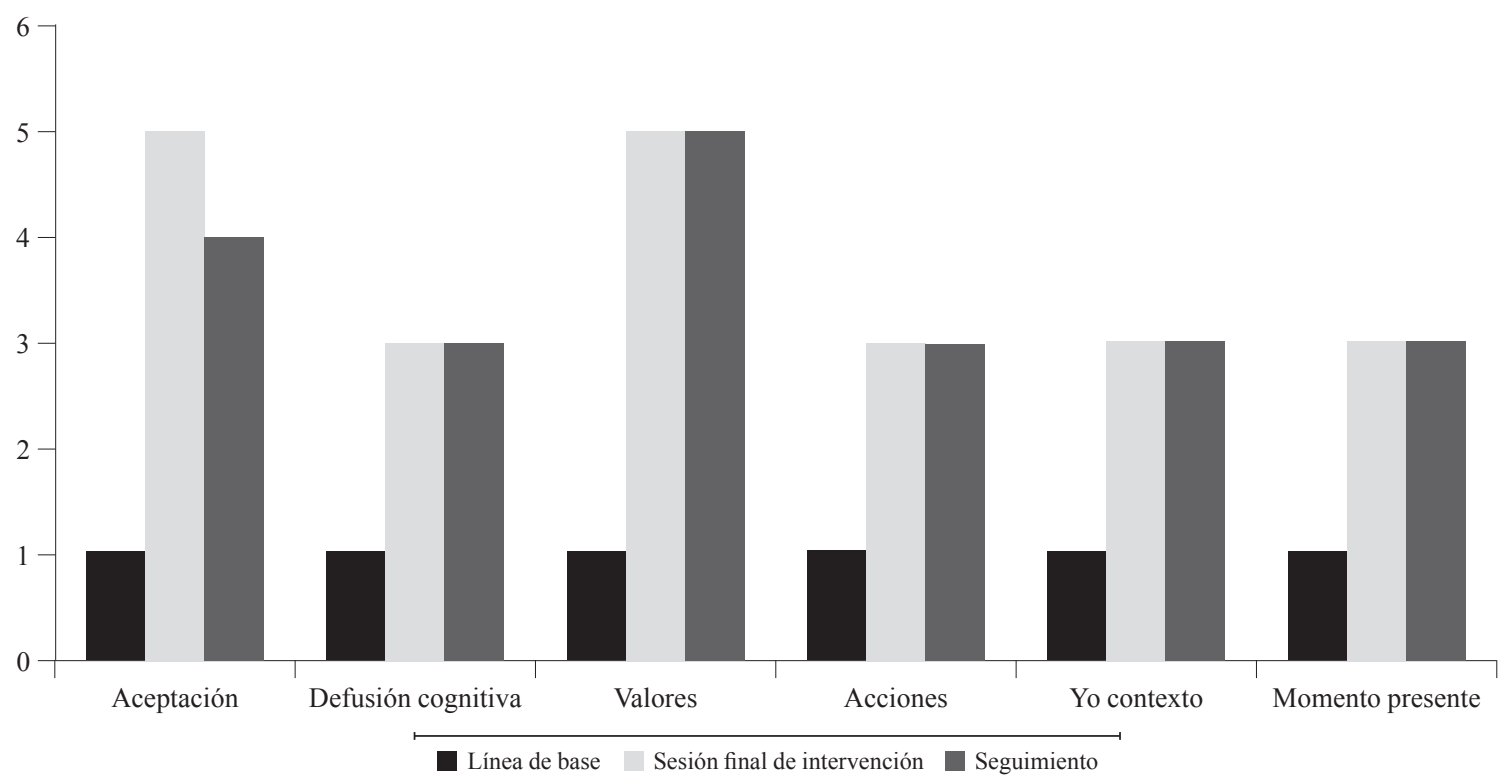

Figura 4. Puntuaciones obtenidas en la línea de base, al final de la intervención y en el seguimiento por la participante 2 en los componentes del Hexaflex 
valores como componente positivamente cambiante, el área de acciones comprometidas descrita en el párrafo anterior mantuvo puntuaciones medias, lo que podría indicar que, aun cuando se generen metas, se podría mantener un problema con el compromiso puntual de acciones hacia estos valores.

\section{Discusión}

Este estudio tenía como principal objetivo indicar desde una aproximación contextualista la efectividad que tiene la implementación de los componentes de la ACT dentro de una intervención orientada a valores en dos mujeres que, a pesar de no ser población clínica, sí presentaban un patrón de evitación experiencial en el fortalecimiento de sus repertorios de flexibilidad psicológica.

Desde el análisis funcional de las participantes, se identificó que las dos presentaban un comportamiento caracterizado por evitar diferentes situaciones que les produjeran malestar, con lo cual, ante esta evitación, configuraban sus reglas verbales, valores y metas en su vida, y que les estaban generando malestar. De esta manera, las participantes desplegaban estrategias similares ante diferentes áreas de su vida (familiar, social y de pareja), y esto correlacionaría positivamente con los resultados de las dos participantes. En la línea de base, tanto en las puntuaciones del Hexaflex como en las del AAQ-II, se encontró rigidez psicológica, a fin de evitar el malestar, desviándose de sus valores y respondiendo la mayor parte del tiempo a sus pensamientos y sentimientos que hacían referencia al momento pasado o al miedo hacia el futuro y no a las contingencias del presente, como lo describieron Valdivia et al. (2010).

Es importante señalar que los componentes evaluados formarían parte de un proceso holístico, y que el trabajo y mejoría en un área repercutiría positivamente en las otras en diferentes magnitudes. Convendría decir que, dada la unificación de los componentes dentro de los repertorios conductuales de las participantes, fue necesario ver cómo todos los componentes trabajaban al mismo tiempo; de esta forma, si uno fallaba, se imposibilitaba el fácil funcionamiento del otro. En este sentido, como lo señala Villate (2014), si una persona presenta un repertorio conductual que obedece a la fusión cognitiva, está más propensa a evitar, a perder el contacto con el presente y verse como un "yo contenido".

Los resultados presentados en este trabajo permiten dar cuenta del objetivo de esta investigación, en relación con el Hexaflex y sus seis componentes. Como indican Dahl et al. (2013), la aceptación como el seguimiento de una regla que especifica el contacto de la función de los estímulos de eventos psicológicos, sin intentar cambiarlos o modificarlos, se integra de manera importante con la defusión cognitiva, ya que esta indica que esa regla que especifica diferentes funciones no está determinada por la literalidad del lenguaje. No hay un control mismo por los pensamientos y emociones $\mathrm{y}$, por consiguiente, se dio lugar a una aceptación de la experiencia. Estos dos componentes se unen en su objetivo al momento de permitir la flexibilidad psicológica de las participantes, ya que el objetivo era permitir la experiencia sin que se dé lugar a la evitación, y para ello era necesario generar repertorios en los cuales no se diera frecuentemente el uso de prejuicios, justificaciones o explicaciones respecto a lo que el lenguaje y su historia indique, sino que esta se permite de una forma no rígida.

En el caso puntual de las dos participantes, los dos procesos aumentan de manera conjunta; sin embargo, el componente de aceptación obtiene, en términos generales, mayor importancia. Esto podría relacionarse positivamente con los ejercicios de mindfulness propuestos en la estructura de intervención, ya que permitirían generar en las participantes la capacidad de vivir el aquí y el ahora sin generar juicios de valor. Esto aumentaría la capacidad de vivir la aceptación y, al mismo tiempo, apuntaría a la experiencia sin juicios de valor, factor que se relaciona con la defusión cognitiva. No obstante, esta se podría relacionar con mayor 
fuerza a la función del lenguaje, el cual tiene mayor resistencia en el proceso terapéutico.

Por esto, podría ser que sus calificaciones fueron un poco más bajas a lo largo del proceso terapéutico, ya que el lenguaje genera relaciones arbitrarias entre los estímulos y llevan al control discriminativo cada vez más refinado, excluyendo así cada vez más propiedades contextuales, que quedan bajo el control del lenguaje (Barnes et al., 2004). Existe una tendencia en las participantes, en especial la 2, por su propia historia de aprendizaje y las variables individuales, a ser insensible a las contingencias por ese mismo control del lenguaje y las relaciones que de él se derivan. Por esta razón, no tiene picos altos en su calificación de defusión cognitiva, en comparación con la participante 1, que sí mantiene una constante en puntuaciones medias-altas (4 puntos). Otro elemento relevante en relación con el poco cambio terapéutico evidenciado en la participante 2 son sus bajas puntuaciones clínicas del pretest (29 puntos) en cuanto al TEE.

Entendido esto, se hace hincapié en la defusión como la principal causa de la inflexibilidad psicológica. En este sentido, los resultados obtenidos por la participante 2 muestran su dificultad para la defusión, pues en la fase de seguimiento estas son las puntuaciones más bajas, lo que correlacionaría, según Villatte (2014), con los bajos niveles de acciones dirigidas a sus valores, ya que "trabajar en acciones requerirá menos esfuerzo si la defusión y los valores son fuertes, ya que las barreras del significado de la vida pueden ser más fácilmente pasadas por alto en este punto" (p. 9), es decir, en la medida en la que se trabaje con los participantes en la defusión psicológica y en la orientación a valores, los otros componentes serán más fáciles de trabajar.

Los anteriores resultados podrían verse como una etapa del cambio psicológico ya que, como señala Villatte (2014), la defusión es el antídoto de la misma fusión; por ello es necesario que los terapeutas acompañen a las participantes durante todo el proceso psicoterapéutico con calidez y apoyo en las historias que contaban, incluso si estas eran desagradables y difíciles de vivir por momentos para ellas.

En relación con los componentes de valores y acción comprometida, Dahl et al. (2013) señalan que los valores son las direcciones generales de la vida de cada participante o sus áreas importantes (familiar, recreación, emocional, social, entre otras) y que las acciones comprometidas son los actos que se toman en dirección a esos valores. De ahí que estos dos componentes se encuentran unidos en la implicación. En relación positiva con los ejercicios propuestos por la estructura de intervención, que tenían gran fuerza en cuanto a los valores y a la acción comprometida, se observa un incremento importante y llega a tener las puntuaciones más altas entre todos los componentes para las dos participantes, es decir, su conducta en sesión y fuera de ella estaba entre un $50 \%$ y un $65 \%$, orientada a la flexibilidad en estas áreas. Esto les permitió a las participantes un aumento en conductas dirigidas a áreas valiosas, en las que se generaron metas en relación con sus necesidades e intereses escogidas libremente; además, sus acciones estaban encaminadas a ellas a pesar de posibles resultados negativos.

Sin embargo, es importante hacer hincapié en los datos obtenidos por la participante 2 , ya que durante las sesiones terapéuticas aumentaron las conductas orientadas a la identificación de áreas importantes de la vida, más que de acciones comprometidas con ellas; por eso el componente de acciones comprometidas en su calificación no tienen un aumento constante junto al componente de valores. Esto posiblemente se puede explicar, porque se reforzó el hecho de verbalizar el deseo de hacer, más que el refuerzo contingente y directo del hacer. Estos resultados pueden deberse a la no generalización de los cambios conductuales dentro de sesión a su ambiente natural y del procedimiento de correspondencia decir-hacer mencionado, ya que los repertorios conductuales pueden cambiar a partir de la conducta verbal, donde se debe reforzar 
diferencialmente la correspondencia decir-hacer y no solo el decir (López, Valverde \& Luciano, 2011).

El yo como contexto y el momento presente son dos componentes dirigidos a permitir a la persona contactar con las contingencias directas; la flexibilidad de este dominio les permitió a las participantes ser conscientes de su propia actividad y cómo esta se relaciona con su experiencia y las variables ambientales (Dahl et al., 2013). En el caso de la participante 1 , debido a variables ideográficas y la aplicación de la estructura de intervención, los ejercicios de mindfulness y el uso de metáforas como la del "autobús y los pasajeros" y "el hombre en el hoyo" permitieron que las calificaciones aumentaran en los dos componentes al mismo tiempo y en igual magnitud a lo largo del proceso terapéutico con una tendencia positiva, lo que se traducía en la capacidad de identificar cuándo se alejaba del momento presente, la facilidad de contactarse con ello mediante instrucciones y poca preocupación por eventos del futuro o del pasado (Hayes, Wi1son, Robinson \& Strohsal, 2014). En el caso de la participante 2 se puede identificar un aumento conjunto en los dos componentes; sin embargo, sus puntuaciones no son tan altas, debido a que en la sesión pierde contacto con las contingencias presentes y con la instrucción dada y queda atrapada en diferentes áreas del contenido de su lenguaje.

En relación con los hallazgos encontrados en el cuestionario AAQ-II, se debe tener en cuenta que las dos participantes, tras el proceso de intervención, aumentaron su percepción de cambio psicológico. En este sentido, podría decirse que el cambio de percepción correlacionó con el cambio conductual del repertorio de inflexibilidad de las participantes descrito, como resultado del plan de intervención propuesto.

Adicionalmente, se encontró una similitud en las participantes respecto a las altas puntuaciones en el primer factor (desbordamiento emocional); además, las dos participantes disminuyeron las percepciones de incapacidad de hacer frente a las responsabilidades y aceptaron sus sentimientos, lo que a su vez se relaciona con las altas puntuaciones en el componente de aceptación del Hexaflex. Tales datos comprueban la confiabilidad y la validez del instrumento para evaluar la evitación experiencial y la aceptación psicológica (Barraca, 2004). De igual forma, en el factor respuestas ineficientes para los problemas de la vida, las participantes tienen puntuaciones bajas, es decir, por un lado, en la fase de evaluación ellas percibían tener respuestas efectivas para sus problemas - en otros términos, las participantes veían que la evitación de sus eventos privados y la no aceptación de ellos era la solución a sus problemas de su vida diaria-, y tras la intervención esta percepción puntúa de forma favorable. Es decir, las participantes lograron entender que la aceptación de sus eventos privados y dejar de luchar con ellos es el mejor camino para solucionar sus problemas (Kohlenberg et al., 2005).

Como punto importante se resalta que en las sesiones se veía el cambio conductual en el momento de introducir las metáforas, ya que las verbalizaciones de las participantes, al inicio, presentaban una marcada inflexibilidad psicológica; pero tras la implementación de la primera metáfora, las verbalizaciones iban cambiando.

Con respecto a las limitaciones de este estudio, es posible que si se hubiera contado con más tiempo de sesiones de intervención, se hubiera observado una mayor modificación en los repertorios de las participantes. Además, si se hubieran implementado autorregistros semanales, posiblemente se tendrían datos para mayor control de las variables intervinientes en sus contextos naturales $y$, de esta manera, usarlas como herramienta factibles para llegar a la generalización de las mejorías que se presentaron en sesión. Finalmente, es importante indicar que con este tipo de investigación no se puede llegar a concluir que el cambio ha sido producido solamente por la intervención de manera clara, teniendo en cuenta las limitaciones del diseño de caso único expuestas, sin influencia de otras amenazas a la validez interna y el seguimiento 
en tan corto tiempo, pues el mantenimiento de los resultados debería medirse a más largo plazo.

El planteamiento de estas dificultades en el proceso puede esbozar positivamente el desarrollo de próximas investigaciones y análisis similares, en las que se tengan en cuenta dichos factores; además de nuevas investigaciones en la aplicación de la ACT de un ambiente terapéutico en el que se tengan en cuenta las interacciones que se dan entre el terapeuta y el participante, ya que esto permitiría comprobar el efecto de la propia intervención con una mayor fiabilidad. Frente a esto, la propuesta de Schoendorff y Steinwachs (2012) es realizar un entrenamiento a terapeutas en ACT en un modelo inspirado en la psicoterapia analítico-funcional, mediante la creación de un contexto funcionalmente similar a la de una relación terapéutica, lo que ayudaría para que el terapeuta preste mayor atención a la relación interpersonal que se presenta en sesión y a las conductas relevantes clínicamente.

Para próximos estudios deberían usarse otros tipos de diseño de caso único como: diseños de tratamientos alternativos o línea base múltiple que permitan un mayor control experimental y que posibiliten comprobar la capacidad del tratamiento de producir cambios psicológicos, en función netamente de la intervención y no de variables extrañas que puedan intervenir o algunos componentes aislados (Ferro \& Vives, 2004).

También la aplicación de nuevos instrumentos como el ACT Matrix, propuesto por Polk y Schoendorff (2014), en los cuales se hace una aproximación a los seis componentes del Hexaflex mediante estrategias sencillas que permiten identificar las acciones de los participantes en diferentes contextos, incluso en el ambiente terapéutico, lo que posibilita el avance científico y la generación de mejores y más eficaces estrategias de aplicación de la ACT.

\section{Referencias}

Bach, P. \& Hayes, S. C. (2002). The use of acceptance and commitment therapy to prevent the rehos- pitalization of psychotic patients: a randomized controlled trial. Journal of Consulting and Clinical Psychology, 70(5), 11-29.

Barnes-Holmes, D. \& Barnes-Holmes, Y. (2000). Relational frame theory and Skinner's verbal behavior: a possible synthesis. The Behavior Analyst, 23(1), 69-84.

Barnes-Holmes, D., Barnes-Holmes, Y., Smeets, P. M., Cullinan, V. \& Leader, G. (2004). Relational frame theory and stimulus equivalence: conceptual and procedural issues. International Journal of Psychology and Psychological Therapy, 4(2) 181-214. Recuperado de http://www. redalyc.org/articulo.oa?id $=56040202$

Barraca, J. (2004). Spanish Adaptation of the Acceptance and Action Questionnaire (AAQ). International Journal of Psychology and psychological Therapy, 4(3), 505-515.

Bond, F. W. \& Hayes, S. C. (2002). ACT at work. Handbook of Brief Cognitive Behaviour Therapy, 117-140.

Congreso de la República (2006, 6 de septiembre). Ley 1090 de 2006: por la cual se reglamenta el ejercicio de la profesión de Psicología, se dicta el Código Deontológico y Bioético y otras disposiciones. Diario Oficial (46.383).

Dahl, J., Stewart, I., Kaplan, J. \& Martell, C. (2013). $A C T \&$ RFT in relationships: Helping clients deepen intimacy and maintain commitments using Acceptance and Commitment Therapy and Relational Frame Theory. Oakland: New Harvinger Publications.

Eifert, G., Forsyth, J., Arch, J. E., Keller, M., \& Langer, D. (2009). Acceptance and Commitment Therapy for Axiety Disorders: three case studies examplifying a unified treatment protocol. $\mathrm{Cog}$ nitive and Behavioral Practice, 16, 368-385.

Ferro, R. \& Vives, M. C. (2004). Análisis de los conceptos efectividad, eficacia y eficiencia en psicología. Panacea, 5, 97-99.

García-Celay, I. M. \& León, O. G. (2005). Sistema de clasificación del método en los informes de investigación en psicología. International 
Journal of Clinical and Health Psychology, 5(1), 115-127.

Gaudiano, B. A. (2009). Methodological comparison of clinical trials of Acceptance and Commitment therapy versus Cognitive Behavior Therapy: matching apples with oranges? Behavior Research and Therapy, 47, 1066-1070.

Hayes, S. C. (2004). Acceptance and commitment therapy, relational frame theory, and the third wave of behavioral and cognitive therapies. Behavior Therapy, 35(4), 639-665.

Hayes, S. C., Luoma, J. B., Bond, F. W., Masuda, A., \& Lillis, J. (2006). Acceptance and commitment therapy: Model, processes and outcomes. Behaviour Research and Therapy, 44(1), 1-25.

Hayes, S. C., Masuda, A., Bissett, R., Luoma, J., \& Guerrero, L. F. (2004). DBT, FAP, and ACT: How empirically oriented are the new behavior therapy technologies? Behavior Therapy, 35(1), 35-54.

Hayes, S. C., Strosahl, K., \& Wilson, K. G. (1999). Acceptance and Commitment Therapy: An experiential approach to behavior change. New York: Guilford Press.

Hayes, S. C., Strosahl, K. D., Bunting, K., Twohig, M., \& Wilson, K. G. (2004). What is Acceptance and Commitment Therapy? In S. C. Hayes \& K. D. Strosahl (Eds.), A practical guide to Acceptance and Commitment Therapy (pp. 1-30). New York: Springer.

Hayes, S. C., Strosahl, K. D., Wilson, K. G., Bissett, R. T., Pistorello, J., Toarmino, D. \& McCurry, S. M. (2004). Measuring experiential avoidance: A preliminary test of a working model. The Psychological Record, 54(4), 553-578.

Hayes, S. C., Wilson, K. D., Gifford, E. V., Follete, V. M. \& Strosahl, K. D. (1996). Experiential avoidance and behavioral disorders: a functional dimensional approach to diagnosis and treatment. Journal of Consulting and Clinical Psychology, 64(6), 1152-1168.

Hayes, S., Wilson, K., Robinson, P. \& Strohsal, K. (2014) Boot camp ACT manual: Acceptance and Commitment Therapy. Contextual change. Reno, Nevada: Context Press.

Kohlenberg, R. J., Hayes, S. C., \& Tsai, M. (1993). Radical behavioral psychotherapy: Two contemporary examples. Clinical Psychology Review, 13(6), 579-592.

Kohlenberg, R. J., Tsai, M., Ferro García, R., Valero Aguayo, L., Fernández Parra, A. \& Virués-Ortega, J. (2005). Psicoterapia analítico-funcional y terapia de aceptación y compromiso: teoría, aplicaciones y continuidad con el análisis del comportamiento. International Journal of Clinical and Health Psychology, 5(2), 349-371.

López, M., Valverde, M., \& Luciano, C. (2011). Contextual control and generalization of say-do correspondence: A preliminary study. International Journal of Psychology \& Psychological Therapy, 11(2), 269-284.

Lozano, M. X. \& Caycedo, C. (2008). Terapia de aceptación y compromiso para un caso de trastorno de evitación experiencial (tesis de especialización). Pontificia Universidad Javeriana, Bogotá, Colombia.

Luciano, C., Gutiérrez, O. \& Rodríguez, M. (2005). Análisis de los contextos verbales en el trastorno de evitación experiencial y en la terapia de aceptación y compromiso. Revista Latinoamericana de Psicología, 37(2), 333-358.

Luciano, C. \& Hayes, S. C. (2001). Trastorno de evitación experiencial. International Journal of Clinical and Health Psychology, 1, 109-157.

Luciano, M. C. \& Valdivia, M. S. (2006). La terapia de aceptación y compromiso (ACT): fundamentos, características y evidencia. Papeles del Psicólogo, 27, 79-91.

Montero, I. \& León, O. G. (2002). Clasificación y descripción de las metodologías de investigación en psicología. Revista Internacional de Psicología Clínica y de la Salud, 2(3), 503-508. Muñoz-Martínez, A. M. \& Novoa-Gómez, M. M. (2010). Análisis de la confiabilidad y validación de un modelo de formulación clínica conduc- 
tual (tesis de maestría). Pontificia Universidad Javeriana, Bogotá, Colombia.

Powers, M. B., Zum Vör de Sive Vörding, M. B., \& Emmelkamp, M. P. (2009). Acceptance and Commitment Therapy: A meta-analytic review. Psychotherapy and Psychosomatics, 78, 73-80.

Polk, K. \& Schoendorff, B. (2014). The ACT Matrix: A new approach to building Psychological flexibility across setting and populations. Oakland: New Harbinger Publications.

Prevedini, A. B., Presti, G., Rabitti, E., Miselli, G., $\&$ Moderato, P. (2011). Acceptance and Commitment Therapy (ACT): The foundation of the therapeutic model and an overview of its contribution to the treatment of patients with chronic physical diseases. Giornale Italiano di Medicina del Lavoro ed Ergonomia, 33(1 Suppl A), A53-A63. Recuperado de http://gimle.fsm. it/33/1s psi/08.pdf

Rey, C. (2008). La terapia de aceptación y compromiso ACT: sus aplicaciones y principales fundamentos conceptuales y metodológicos. Revista Suma Psicológica, 11(2), 267-284.

Ribes, E., Torres, C., \& Ramírez, L. (1996). Efecto de los modos de descripción en la adquisición y transferencia de una discriminación condicional de segundo orden en humanos adultos. Acta Comportamentalia: Revista Latina de Análisis del Comportamiento, 4(2), 159-179.

Russo, A. \& Forsyth, J. (10 de abril de 2013). Psyciencia. Recuperado de http://psyciencia. com/2013/04/10/una-introduccion-a-terapia-de-aceptacion-y-compromiso/

Roussos, A. J. (2007). El diseño de caso único en investigación en psicología clínica: un vínculo entre la investigación y la práctica clínica. $R e-$ vista Argentina de Clínica Psicológica, 16(3), 261-270.

Ruiz, F. J. (2010). A review of Acceptance and Commitment Therapy $(A C T)$ empirical evidence: Correlational, experimental psychopathology, component and outcome studies. International
Journal of Psychology and Psychological Therapy, $10,125-162$.

Schoendorff, B. \& Steinwachs, J. (2012). Using Funtional Analytic to train therapists in Acceptance and Commitment Therapy, a conceptual and practical framework. International Journal of Behavioral Consultation and Therapy, 7(2-3), 137-139.

Suárez-Aguirre, J., Luciano, C. \& Ruiz, F. (2010). Impacto de un protocolo basado en aceptación en el rendimiento cognitivo en una tarea experimental: un estudio piloto. Avances en Psicología Clínica, 160, 237-240.

Torneke, N., Barnes-Holmes, D. \& Hayes, S. (2010). Learning RFT. Oakland: New Harbinger Publications

Torneke, N., Luciano, C. \& Valdivia, S. (2008). Comportamiento gobernado por reglas y problemas psicológicos. International Journal of Psychology and Psychological Therapy, 8(2), 141-156.

Valdivia, S., Sheppard, S. \& Forsyth, J. (2010). Acceptance and commitmenr therapy in an emotional regulation context. En A. Kring \& D. Sloan (Eds.), Emotional regulation and psychopathology: a transdiagnostic approach to etiology and treatment (pp. 301-338). New York: Guilford Publications.

Valero Aguayo, L. \& Luciano Soriano, M. C. (1992). Relaciones de equivalencia: una síntesis teórica y los datos empíricos a nivel básico y aplicado. Psicothema, 4(2), 413-428.

Villatte, M. (2014). Defusion through infusion: practice ground. Recuperado de https://www.practiceground.org/blog/2014/04/defusion-through-infusion/

Wilson, K. G. (2007). The Hexaflex diagnostic: A fully dimensional approach to assessment, treatment, and case conceptualization. Presidential address presented at the annual convention of the Association for Contextual Behavioral Science, Houston, TX.

Wilson, K. G., Bordieri, M., Flynn, M. K., Lucas, N., \& Slater, R. (2010). Understanding acceptance 
and commitment therapy in context: A history of similarities and differences with other cognitive behavior therapies. En J. Herbert \& E. Forman (Eds.), Acceptance and mindfulness in cognitive behavior therapy. Hoboken, NJ: Wiley.
Wilson, K. G. \& Luciano, C. (2002). Terapia de aceptación y compromiso (ACT): un tratamiento conductual orientado a los valores. Madrid: Pirámide. 